\title{
Immersion and Togetherness: How Live Visualization of Audience Engagement Can Enhance Music Events
}

\author{
Najereh Shirzadian ${ }^{1}$, Judith A. Redi ${ }^{2}$, Thomas Röggla ${ }^{1}$, Alice Panza ${ }^{1}$, Frank \\ Nack $^{3}$, Pablo Cesar ${ }^{1,2}$ \\ ${ }^{1}$ Centrum Wiskunde \& Informatica \\ Science Park 123, Amsterdam (NL) \\ ${ }^{2}$ Delft University of Technology \\ Mekelweg 2, Delft (NL) \\ ${ }^{3}$ University of Amsterdam \\ Science Park 904, Amsterdam (NL)
}

\begin{abstract}
This paper evaluates the influence of an additional visual aesthetic layer on the experience of concert goers during a live event. The additional visual layer incorporates musical features as well as biosensing data collected during the concert, which is coordinated by our audience engagement monitoring technology. This technology was used during a real Jazz concert. The collected measurements were used in an experiment with 32 participants, where two different forms of visualization were compared: one factoring in music amplitude, audience engagement collected by the sensors and the dynamic atmosphere of the event, the other one purely relying on the beat of the music. The findings indicate that the visual layer could add value to the experience if used during a live concert, providing a higher level of immersion and feeling of togetherness among the audience.
\end{abstract}

\section{Introduction}

This paper evaluates if and how a real-time live visualization adds value to the experience of concert goers. We have developed an additional visual aesthetic layer on top of the music, coordinated by our audience engagement monitoring technology using GSR (Galvanic Skin Response) sensors. Our wearable biosensing technology provides a reliable, fine-grained, and continuous mechanism to quantify the experience of people. This measurement technology was used during a real concert organized by a well known (international) institution that periodically hosts a series of Jazz events. The measurements were used during a lab experiment where we tested two visualizations on 32 participants. One of which factoring in the level of audience engagement collected by the sensors, the other one purely relying on the beat and amplitude of the music. The results indicate that the visual layer could add value to the concert experience, 
providing a higher level of immersion and feeling of togetherness among people. Thus, the final visualization combines the music amplitude, the level of audience engagement collected using the GSR sensors and the dynamic atmosphere of the event.

Enriching a music event with visual content is an art field in itself which began in the late 1980s and has since been further developed worldwide by video artists, the so-called 'VJs' [29,12]. As technology progresses, opportunities for making musical events even richer are blooming. Experiments have shown how combining music with visuals, but also light and even smell, can provide the audience with an enhanced experience. Dekker refers to these performances as synaesthetic performances, synthesizing various media and therefore creating a physical and psychological connection with the public [6]. User generated content has also played an important role in this development [10]. For example, Engstrm et al. [8] have proposed to bring the trend of collaborating on media content into the nightclub scene. An app was designed that allows users to directly upload videos to the VJ following requirements gathered with artists and audience.

Traditionally, performances are visually enriched by coordinating and visualizing the active participation of the audience, involving them in the creation process $[4,15,30]$. Our work instead explores a more transparent and less intrusive approach: real-time monitoring of audience engagement based on wearable sensors. Wearable sensors have recently become a reliable source of information about the affective state of people, and are commercially used for several distinctive applications such as well-being and gaming [22,23]. Among wearable sensors, the most relevant for monitoring audience engagement are those capable of tracking physiological signals such as Heart Rate or Galvanic Skin Response (GSR). Especially the latter has been shown in a number of studies to correlate with user arousal $[18,19,31]$, i.e. the level of activation of a person [25]. As a consequence, GSR signals have been used in literature as a proxy measure for engagement, which we use as the basis of our proposed visualization enriching the experience for concert goers.

We leverage the power of sensing the state of crowds attending a concert through physiological sensors. Such a real-time source of data is then used for creating a visualization that enriches the actual concert, triggering higher immersion and feeling of togetherness within the audience. Based on interviews with professionals and audience members, we gathered a number of requirements for developing the visualization.

At the aesthetic level, the artistic goals were to create a 'collective artwork', which dynamically transforms based on the level of engagement of the crowd. Still, the visualization was kept abstract enough, so no direct conclusion regarding the quality of the music could be drawn, which could in turn negatively affect the musicians or the crowd. Finally, audience members can identify themselves if they would feel like it, providing extra awareness about his/her state and his/her relationship with others at the event.

From a more scientific point of view, we hypothesize that by augmenting a live event with a visualization of the audience engagement, we deliver a richer 
experience. In particular, we propose a new visualization approach that extends existing models based only on music, taking into consideration the atmosphere of the event. The overlap of these two sources of information makes the audience experience a multi-layered event, which combines music and higher awareness of the audience engagement. As a result, we define our research question as: Does the integration of user engagement information add value to the user experience of a live musical event in terms of increased immersion and togetherness?

In the remainder of this paper we further report our efforts, using mixedmethod methodologies: interviews with professionals and audience members, an experiment in which GSR data was collected from a relatively large crowd at a live Jazz concert, and a second experiment in which 32 audience members evaluated the visualization based on immersion and togetherness in a lab setting. Section 2 examines previous research in the fields of visual enrichment of music events, measurement approaches of audience engagement, immersion and togetherness. Section 3 describes the mixed-methodology approach used, and the rich data set collected during the various experiences. Section 4 describes the developed visualization and Section 5 reports the results. Finally, Section 6 provides an analysis of the results and a discussion of these.

\section{Related Work}

\subsection{Visual Enrichment of a Concert}

Music visualization has a big role in the artistic scene, dating back to the late 1980s. So called 'video jockeys' or 'VJs' are video artists usually working in live performances, complementing music with visuals [6]. While the working field for VJs expands also towards museums, art galleries, live shows and concerts, the main field still remains the nightclub scene [9]. Still, the development of user centered media reveals new opportunities in visual enrichment of music events. Engstrm et al. state that the importance of user generated content is increasing when it comes to the production of hybrid media [10]. In a 2008 study, they investigated new ways for the audience to contribute to live visuals, using a mobile app to upload videos directly to the VJ. The experiment showed that this increased participation to the visuals triggered a new collective experience [8]. In addition, it is known that, as social beings, we are strongly affected by the opinion (or affective state) of others around us, them being virtually or spatially collocated [13].

This leads us to hypothesize that enriching visualization with data reflecting the audience affective state may further increase the user's feeling of participation and immersion in the event.

\subsection{Sensing User Engagement}

Engagement has been defined by Attfield as 'a quality of the user experience that emphasizes the positive aspects of interaction in particular the fact of 
being captivated by a resource' [2], p.9, and similarly described as a state of high affective involvement of a user with an experience [28].

Previous work has identified a number of ways for monitoring the level of engagement of people with experiences, but those are mostly based on selfreport [21]. Gathering data during a live concert puts practical constraints on the measurement, since it should not affect the users' experience of the event. In this regard, wearable sensors offer a valuable alternative to self-reported data [20]: being attached to the user skin in a relatively unobtrusive way, they can gather measurements of the affective state of the users without harming the user experience. Lang found a linear correlation between GSR and human arousal [18]. Building on these results, a number of studies have leveraged the power of GSR sensors to better understand user experiences during live performances.

Wang et al. measured the GSR response of a group of test users during a live performance which was also recorded on video. Later, the video recording was compared with the data gathered from the sensors and results from questionnaires and interviews: the researchers validated that GSR sensors are an accurate proxy for measuring audience engagement [31]. Latulipe also supports the approach of interpreting GSR signals as audience engagement after running an empirical study with 49 participants. A video of a dance performance was presented to the participants, who were equipped with GSR sensors and scales that allowed them to self-report their current state of emotional reaction [19]. The two measurements were found to be correlated.

At this stage, it is important to mention that arousal itself may not be sufficient to characterize engagement: an indication of the positivity of the state (valence) would be missing. Since the GSR sensors can only measure arousal, and not valence, it is not possible to state if the experienced emotion is positive or negative. In this regard, Latulipe ran an exploratory study, where she showed audience engagement data of performances to performance arts experts. These experts stated that without a causal explanation, the valence factor would not be interesting anyway, since valence is a very subjective variable [19]. Based on this result, we propose in this paper to use GSR as a proxy measurement for arousal, being well aware of the need of furthering this research in the future to include valence measurements in the visualizations.

\subsection{Enriched Performances: Immersion and Togetherness}

Previous works have considered a number of approaches to provide feedback to musicians, actors, and producers. Some examples include off-line visualization tools [24], real-time mechanisms such as mobile phone usage [30] and messages [4], and audience movement [15]. We nevertheless explore audience engagement as the basic element for creating the visualizations. In particular, we explore the effect of the visualization on the level of immersion and togetherness of the crowd.

Immersion is an aspect which occurs in gaming, as well as virtual environments, but also in visiting art exhibitions or watching movies. Jennett et al. state that immersion consists of three features [16]: (1) Lack of awareness of time; (2) 
Loss of awareness of the real world and (3) Involvement and sense of being in the task environment. Several measurement questionnaires have been developed and tested. Witmer et al. define immersion and involvement as two important aspects for experiencing presence. They introduced a 'presence questionnaire' to measure presence in virtual environments [32]. Jennett et al. developed a questionnaire to measure immersion in games [16]. They base their definition of immersion on two descriptive studies of Brown and Cairns and Haywood and Cairns. In the first study, 'gamers' were interviewed about their experience in playing computer games [3], the second study dealt with children in an interactive exhibition [14].

Along with immersion, we are also interested in increasing the sense of togetherness in the audience. In our research, the aspect of 'togetherness' is equated to the degree of 'feeling part of a group'. In a music show, the audience member can feel as part of the audience, but also - when included with GSR data in the visualization - part of the concert/show. Previous works describe different approaches to measure the feeling of being part of a group. The 'group attitude scale' (GAS) is a measurement tool with 20 items, which was developed to measure attraction to a group. The selected items were tested in several studies and provide a valid measure of attraction to groups [11]. Besides text-based measurement tools, there are also graphical measurement options to get an insight in to what degree a person feels part of a group. Schubert et al. developed a pictorial scale of 'Ingroup-Outgroup Overlap' and 'Self-Group Overlap' (OSIO) [27], building up on the 'inclusion of others in self' (IOS) scale of Aron et al. [1]. The tested and proven OSIO measurement scales are easy to use and well understood.

\section{Methodology and Data Collection}

This research followed a mixed-methodology approach: both requirement gathering and evaluation were done using qualitative and quantitative mechanisms. First, requirements were gathered through interviews with musicians and concert organizers. Then, GSR data was gathered from the audience of a live concert. Requirements from the audience perspective were also gathered through questionnaires. According to these requirements, visualizations were created, which were evaluated quantitatively in a second experiment with potential audience members. Additionally, a qualitative evaluation was conducted with the musicians and the concert organizer, as described in Section 5. The real concert, which we based our requirements on, was organized by a cultural institute in a major European capital.

\subsection{Requirement Gathering with Performers and Event Organizers}

Initial interviews provided first insights and information about the requirements and expectations of the visualization. A concert organizer and the musicians were interviewed in a semi-structured way in order to gain more knowledge about what the persons in different roles would expect from a visualization that 
shows the audience's engagement. The interviews with the concert organizer and the musicians were held in person or on the phone, recorded and transcribed.

Insights gained from the interviews showed that sensing and feeling the audience is very important for the musicians interviewed.

"Otherwise I would only record CDs. If you enter the stage, you already feel the atmosphere, so you know what the vibe is a little bit." - Musician 1.

Both musicians pointed out that a live visualization during the concert would not be valuable for them. In part, that was because their eyes are closed during playing; partly it was because - should the visualization show low audience engagement - they would feel insecure about their play and get influenced by the visualization. Still, both musicians mentioned, if the visualization would show a very highly engaged audience, the visualization would influence them in a positive way and strengthen them in their play. In general, the musicians saw the potential of a live visualization more for the audience than for themselves:

"Maybe for the audience it would be better. Maybe it should be projected behind you and then the audience can see. Because I think this is really interesting. Maybe it even creates a feeling of collectiveness." - Musician 1.

The perspective of the concert organizer followed this direction. A live visualization of the audience engagement would not be used for evaluations of the event.

"You cannot evaluate art, put it into numbers" - Concert Organizer.

Rather, the concert organizer saw this concept as a new way to form a collective artwork, giving the concert an added artistic visual value. Understanding the concerns of the artists that the visualization might make them feel insecure, the concert organizer pointed out clearly that it was crucial for the event that the musicians would feel at ease and had their space to perform their art, without being disturbed. In addition, a live visualization of the audience engagement would have to be coordinated very closely to the choreography of the performing artist, to create harmony in the performance.

\subsection{Requirement Gathering with Concert Audience}

To gather audience requirements, 40 concert attendees were given questionnaires after the concert. The concert attendees were a mixed crowd of students and culturally interested people between 19 and 70 years, mixed in gender (15 male, 25 female) and mainly based in the city where the experiment was held. Attendees' expectations for future audience engagement visualizations were investigated through a set of 12 questions investigating (1) their experience with the GSR sensors during the concert (see Section 3.4); (2) their interest in seeing live data visualized during the concert and which added value they would find in it and 


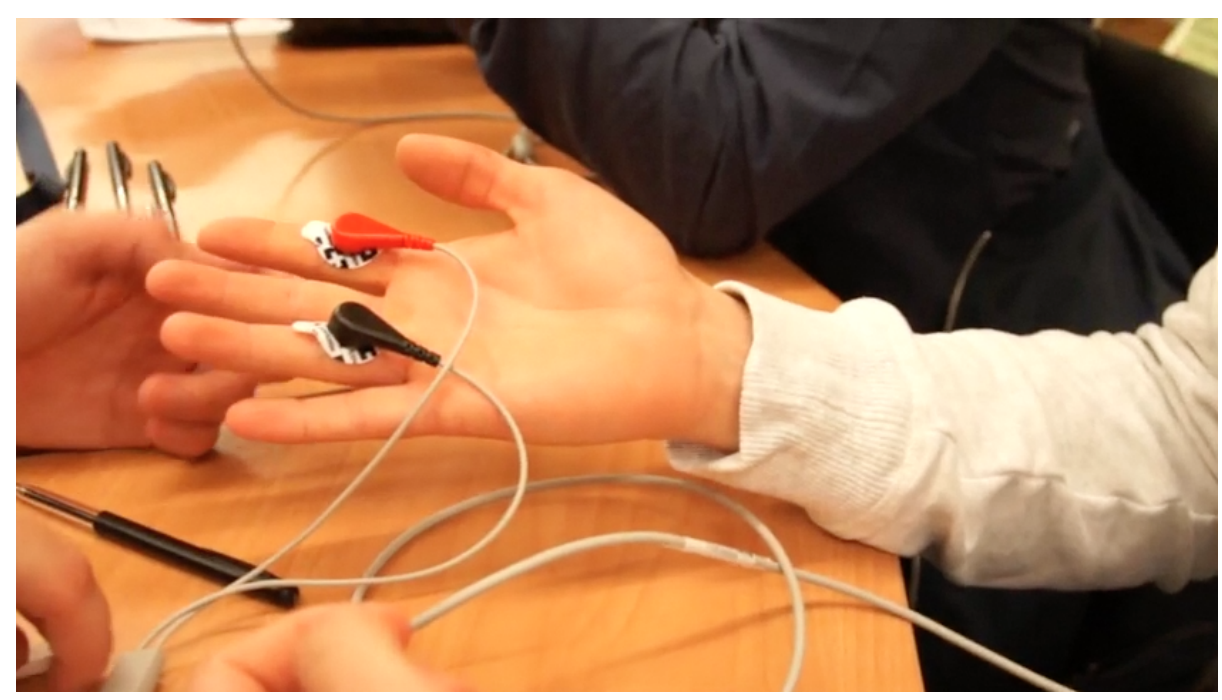

Fig. 1. Audience member with GSR sensor

(3) privacy concerns. All questions were formulated as statements and attendees were asked to indicate their level of agreement with them on Likert scales from 1 to 5 , ( 1 = 'I fully disagree', $5=$ 'I fully agree'). A yes/no question also investigated whether they believed that a live visualization could influence their concert experience.

Results from the questionnaires showed that the users were very interested in the gathered data (mean rating of 4.55) and also very interested in a live visualization during a concert (mean of 4.075). The users were interested in the general mood of the audience (mean of 3.8), but still wanted to be able to identify themselves in the visualization (mean of 3.7). Privacy concerns were voted as not very high (mean rating of 2.1). 77 percent of the attendees stated that a live visualization would influence their concert experience. In comparison, whether the visualization would enrich the experience or distract them from the music, the users were quite indecisive. Both mean values are found in the middle of the scale, while the users tend to say that the visualization would rather distract from the music (mean: 3.2), than enrich the experience (mean: 2.85).

When asked whether visualizations would influence their concert experience, audience members were consistent with the impression of the musicians (see Section 3.1):

"If everyone seemed to be enjoying themselves it might make me feel better. If everyone seemed bored, however, it may make me feel bored too"

"I might get carried away with the general vibe even if it's not my own personal experience"

"Probably I would be influenced by the opinion/feeling of the other people so I would be distracted" 
Finally, an unexpected feature appeared while scanning the questionnaire results: Some audience members saw the potential of 'gamification' in the visualized data, wanting to try to influence their engagement values:

"It would be a great sideshow for those not engaged by the main show. Also a lot of people would probably try to see if they could influence the visualization/compete for who could set the highest GSR"

\subsection{Summary of the Requirements}

The requirements were gathered through semi-structured interviews and questionnaires with both professionals and concert goers. The most important findings that drove the development work, were that the visualization should be intended for the audience and not necessarily for the musicians and that the audience rated the privacy concerns low, while being very interested in the physiological data and its visualization.

\subsection{GSR Data Collection}

The quantitative requirement gathering was set up with the audience of the concert. The concert was acoustically and visually recorded and the recordings were temporally aligned. During the concert, the engagement of 40 users was gathered with GSR sensors and stored (see Figure 1). This was achieved by setting up two Raspberry Pis running specialized software, which acted as receivers for the wireless sensors. Each one of them was responsible for capturing the data sent out by one of the groups of sensors. This data was then forwarded to a laptop which acted as a central hub, processing and storing the data (see Figure 2).

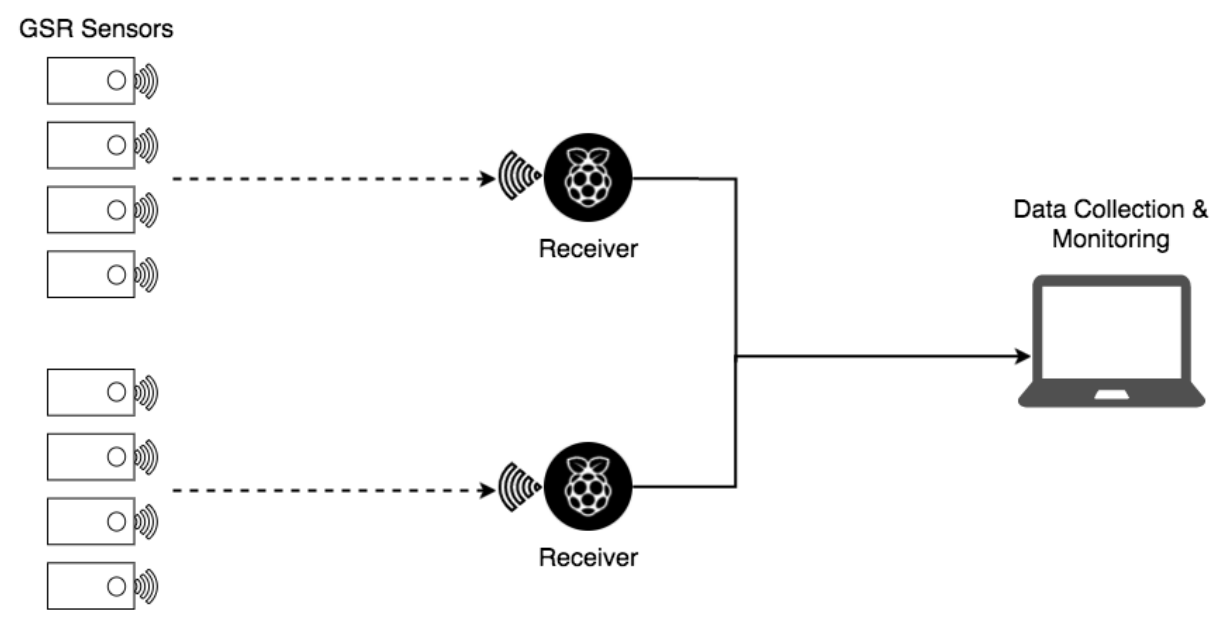

Fig. 2. Network architecture for collecting GSR data streams during the Jazz concert 
In order to collect the data, we deployed an optimized network infrastructure and a monitoring system at the venue (see Figure 3) which could handled large volumes of real-time data from the wearable sensors. Hence, it was possible to collect data from several users at the same time. The entire recording process was synchronized and controlled and the GSR measurements were sent to the receivers through wireless transmission.

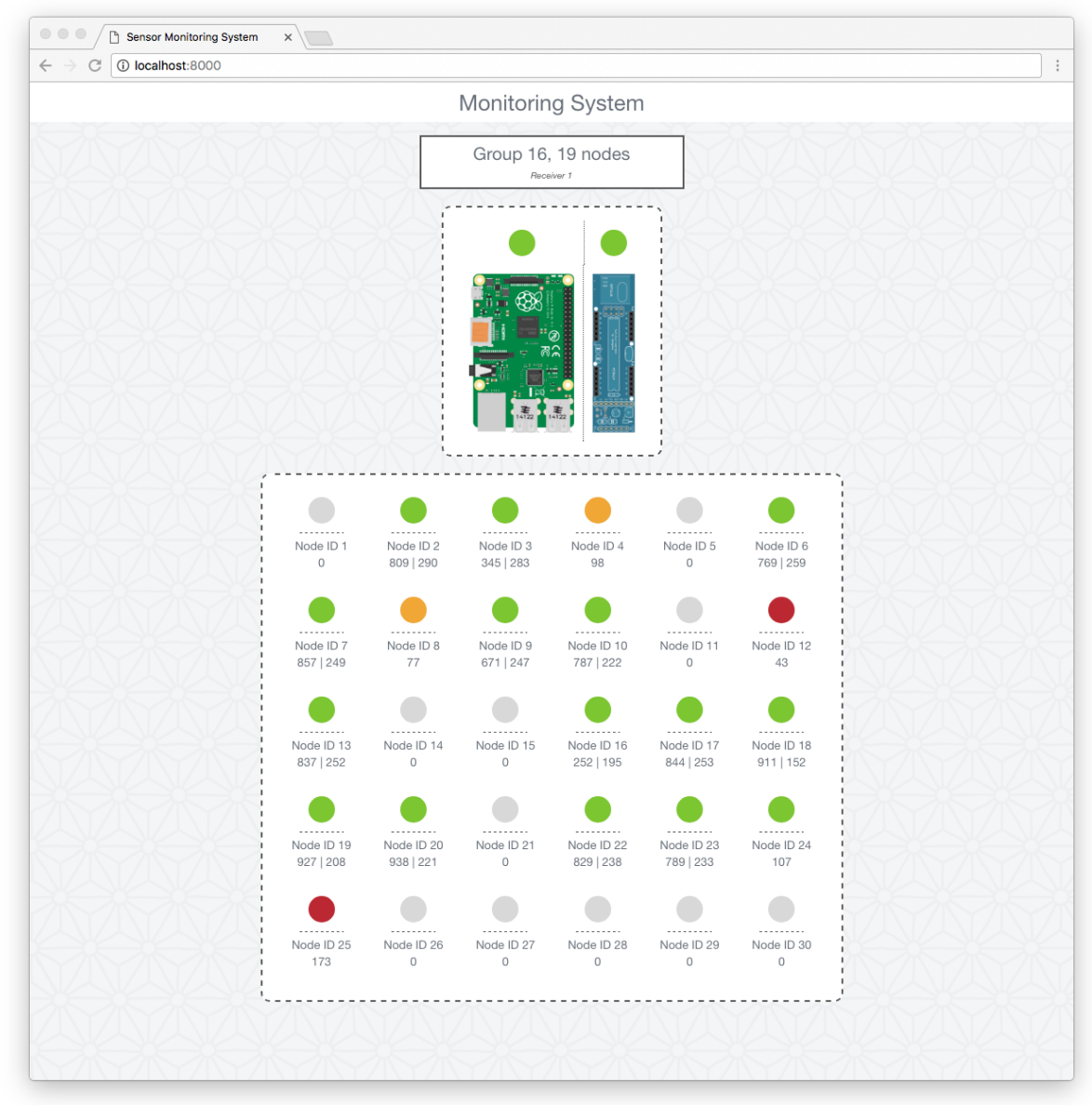

Fig. 3. Network monitoring software, controlling the state of the sensors during the Jazz concert

After the concert, the collected data was run through an algorithm to rid it of artifacts introduced, for instance, by the electrodes temporarily losing contact to a participant's skin, or data packets getting lost in transit to the receivers. As a first step, the data for each sensor were then aligned to the general timeline with 
a granularity of 1 second. As a consequence, if the receiver had received more than one GSR sample for a specific sensor within one second, it would take the average value of those samples. On the other hand, if the receiver did not receive any data from a sensor during a specific second, the value was interpolated from the two adjacent values in the timeline. Finally, the resulting file was analyzed for artifacts. These artifacts manifest themselves through sudden vertical jumps in a sensor's GSR value from one second to the next. This was fixed by differentiating the data for each sensor and zeroing all entries for which the derivative was larger than a certain value. The data was then integrated numerically using a cumulative sum to restore the original signal without the artifacts. Empirically, we found that zeroing values which were more than four standard deviations away from the mean for a given sensor provided a robust way of removing the artifacts without impacting the original signal. A similar process to remove these kind of artifacts was followed by Kocielnik et al. [17] in their experiments.

\section{Creation of the Visualizations}

Based on the requirements gathered from the concert organizer, the musicians and the audience, and on the collected GSR data, two visualizations were created for comparison purposes using Processing ${ }^{1}$, a programming language that allows for the creation of visualizations based on different input types. While one visualization just takes the music as an input, the second visualization takes two parameters as input: the music and the data gathered from the sensors.

The main objective was to create a 'collective artwork', based on the changes of the GSR data in order to show that the audience is triggering and influencing the visualization. We opted for a dynamic visualization, from which conclusions regarding the quality of the music could not be drawn, as directly requested by the musicians and the event organizer. In addition, we wanted to meet the requirements of the audience members to identify themselves in the visualization. Thus, each sensor (audience member) was shown individually as a circle, that moved along the y-axis of the screen according to the value of engagement (as gathered by the GSR sensor). The radius of the circle responded to the amplitude of the music. Additionally, the circles were plotted in different colors, to make the identification more evident. In this example, five colors were used, so each four circles were plotted in the same color. The audience member with the sensor 'blue2' could identify herself/himself as the second blue circle on the screen.

Even though the requirement collection phase showed that the audience was interested in getting information about its 'average mood' in a live visualization, the decision was made not to show the relative/absolute average value of the GSR signals explicitly. Given that the circles represented individual audience members, having a reference 'average mood' indicator, such as a line crossing the visualization horizontally, would have made it possible for the audience to detect how many and which people were currently less engaged than the average.

\footnotetext{
$\overline{1}$ https://processing.org
} 


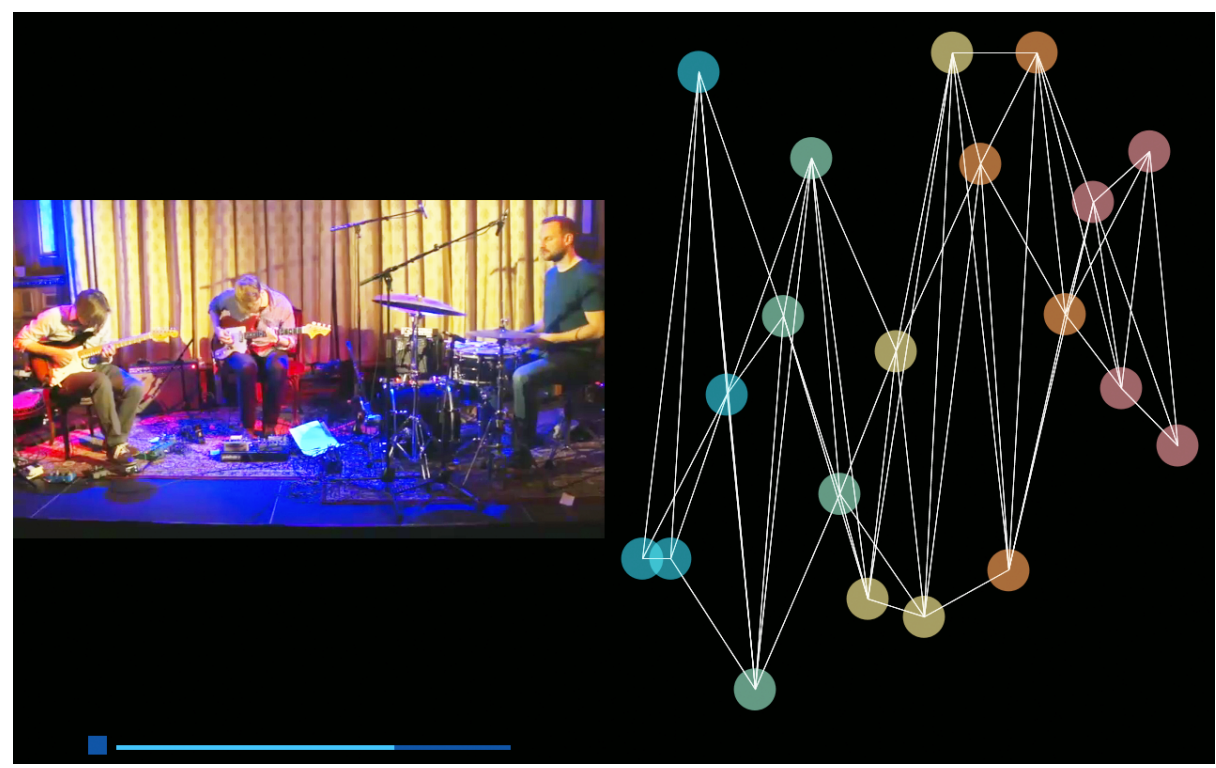

Fig. 4. left side: video recording; right side: visualization with absolute values

Therefore, the final visualization only showed the audience members as circles, floating over the screen, driven by their GSR data, changing size with the amplitude of the music. Still, the connection of the audience was important for the concept of the visualization. Therefore, the audience members were connected to each other with lines. The movement of the audience member pulled the lines with them.

By analyzing the GSR data manually, it was also discovered that the values of the GSR changed gradually, but very slowly. During a concert lasting for one hour, there was movement visible, but it was hardly recognizable for the viewer. To intensify the movement of the visualization, we decided to represent relative changes in engagement with respect to each individual's range of engagement change. For each person, the personal minimum and maximum values were stored, then mapped from the bottom of the screen to the height of the screen (see Figure 4). Every person's circle floated in its own range of minimum and maximum values, representing each person's individual/relative experience and reaction to the music. In addition, to increase the visual focus on movement, the new position of the circle was updated every 5 seconds.

Eventually, an abstract, constantly changing, collective artwork was created, triggered by the feelings of the audience and the music. Even though the 'general mood' of the audience was not explicitly shown with a line anymore, the constant movement and re-formation of the abstract visualization could be understood as the 'general mood' of the room. 


\section{Evaluation of the Visualizations}

To eventually answer our research question:

whether enriching visualizations with audience engagement data during a concert would increase the audience immersion and feeling of togetherness in the event

we performed a controlled experiment. The visualizations described in Section 4 were implemented for excerpts of the concert during which GSR data were collected. They were then displayed alongside the video of the corresponding concert excerpt in a split-screen fashion (see Figure 4, showing the concert recordings on the left side and the visualization on the right side). A time-line in the bottom left corner of the screen allowed to jump to different parts of the show.

Music excerpts were played along with their corresponding visualizations (with and without sensor data) to 32 of participants in a laboratory setting. Although we recognize that this approach has limited ecological validity (as the visualizations were not shown live during an event), we opted for the controlled setting to be able to collect reliable quantitative data. In addition, following our original mixed methodology, a qualitative evaluation was also performed through semi-structured interviews with the concert organizer in person and one of the musicians on the phone. The interview with the concert organizer was recorded and transcribed. Due to technical issues, the recording of the musician failed, therefore the key concepts extracted from the interview, were summed up and confirmed with the musician via e-mail.

\subsection{Experimental Design}

We conducted a full-factorial quantitative evaluation with 32 potential concert visitors. Participants were mainly students, researchers and young professionals, assembled in opportunist sampling fashion. The main factor under investigation was the presence of engagement data (through GSR) within the visualization. We created two visualizations: one as described in Section 4, and the other very similar, but not reacting to sensor data. A second issue was whether the effect of including GSR data in the visualization would depend on the level of 'energy' of the music piece that was being performed. A calmer music piece may have generated limited changes in audience arousal, in turn creating minimum added value in the visualization. Thus, to be able to generalize our results, we created visualizations for two different concert excerpts: a calmer one, and a more energetic one. Finally, because of the complexity of the task (i.e., evaluating music visualizations), we were forced to provide our participants with context about the research, and mention that they would see music visualizations that do or do not react on sensor data. One possible issue there may have been that the sole knowledge of the presence of sensor data in the visualization may have biased the participants. To control for this, for each video and visualization (with and without sensor data) we performed two repetitions, one in which the participant was told that sensor data were visualized, and one in which 
the participant was told that only music was being visualized. We combined the effects of the three factors (visualization of sensor data, energy of the music piece played, and communication of the presence of sensor data in the visualization) in a full-factorial design, for a total of $2 \times 2 \times 2=8$ conditions (see Figure 5).

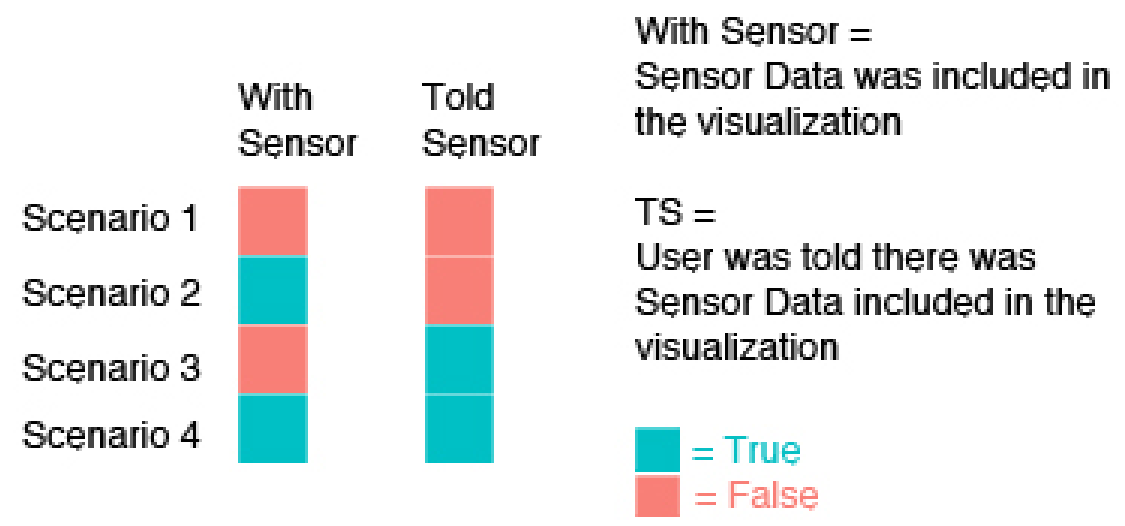

Fig. 5. Full-Factor experiment setup

Measurements. We developed a short questionnaire to evaluate the feeling of Immersion and Togetherness of participants in presence of the visualizations. The questionnaire included four items to measure immersion adapted from Zhu et al. [33], and Schubert et al. [26], and three items to measure togetherness, adapted from previous research of the 'group attitude scale' [11] and 'assessment of Self-Group Overlap' [27] (see Table 1). In addition, participants were asked to report the extent to which they appreciated the music piece played in the video, on a 5 -point Likert scale.

Protocol. Participants were seated in front of a screen. The experiment was held with either three or 4 participants at the same time. Prior steps of the research were explained beforehand. Participants were asked to imagine themselves in the audience of the Jazz concert, wearing the GSR sensors, while seeing the visualizations projected behind the band. Participants were shown two excerpts $(1: 15 \mathrm{~min})$ of the recorded concert, enriched with the two visualizations. Each excerpt was tested in a full-factor procedure. One excerpt was chosen from a calm play, the other one from a rhythmic, energetic part of the concert. In total, each participant watched eight video clips (the reader can find them here: http://goo.gl/2BL0rL), each lasting 75 seconds. The order of the clips was randomized to prevent an influence of fatigue and memory on the results. After each video clip, participants were asked to fill in the questionnaire to self-report their level of immersion and togetherness. 


\begin{tabular}{|l|l|l|l|}
\hline Aspect & Nr. items & Based on & Measure scale \\
\hline Immersion & 4 & {$[33],[26],[11]$} & 5-point Likert \\
\hline Togetherness & 1 & {$[11]$} & 5-point Likert \\
\hline Togetherness & 2 & {$[27]$} & OSIO \\
\hline Liked Music & 1 & - & 5-point Likert \\
\hline
\end{tabular}

Table 1. Questionnaire for the evaluation of the visualization

\subsection{Results: Quantitative Analysis}

We first analyzed the internal consistency of the questionnaire for the Immersion and the Togetherness items separately. For the four Immersion items, we obtained a Chronbach's alpha of 0.879 (excellent internal consistency). We could then conclude that the four items were measuring the same underlying construct, and thus they could be combined (summed) into a single dependent variable hereafter referred to as 'Immersion'. Similarly, for the three togetherness items we found a value of alpha of 0.75 (very good). The three items were then combined into a single variable hereafter referred to as 'Togetherness'.

To verify whether our three fixed factors (visualization of sensor data, energy level of the video and communication of whether sensor data were visualized or not) had an effect on Immersion and Togetherness, we resorted to Linear Mixed Effect (LME) models. LMEs extend classic linear models to properly model data obtained from repeated measures designs (such as ours, where all subjects evaluated all experimental conditions). LMEs allow modelling the repeated measures factors (in our case, the participants) as random effects in the model, thereby accounting for individual differences in the visualization preferences. In effect, a different intercept is estimated per participant, consequently modelling individual participant biases in the Immersion and Togetherness evaluations.

We investigate the impact of the three fixed factors on Immersion and Togetherness through the likelihood ratio testing [5]. We fit first a minimal (null) model including only the random effect (participants) as a predictor. We then iteratively add a term to it for each of the three fixed factors (extended models). Whenever an extended model is found to fit the data significantly better (as evaluated through an ANOVA) than the null model or than a model accounting for a lower number of fixed factors, the newly added factor is considered to have a significant effect on the dependent variable.

With respect to Immersion, we found both the energy level of the concert excerpt $(p=0.005)$ and the visualization of sensor data $(p<0.001)$ to have a significant effect. A model including both factors also performed better than models including only either of them. The fact that participants were told or not that sensor data were included in the visualization did not have a significant effect $(p=0.69)$. These findings can be visually inspected in Figure 6$)$. Clearly, 
the visualization of sensor data has the biggest effect on immersion, while the impact of the level of energy of the video is smaller.

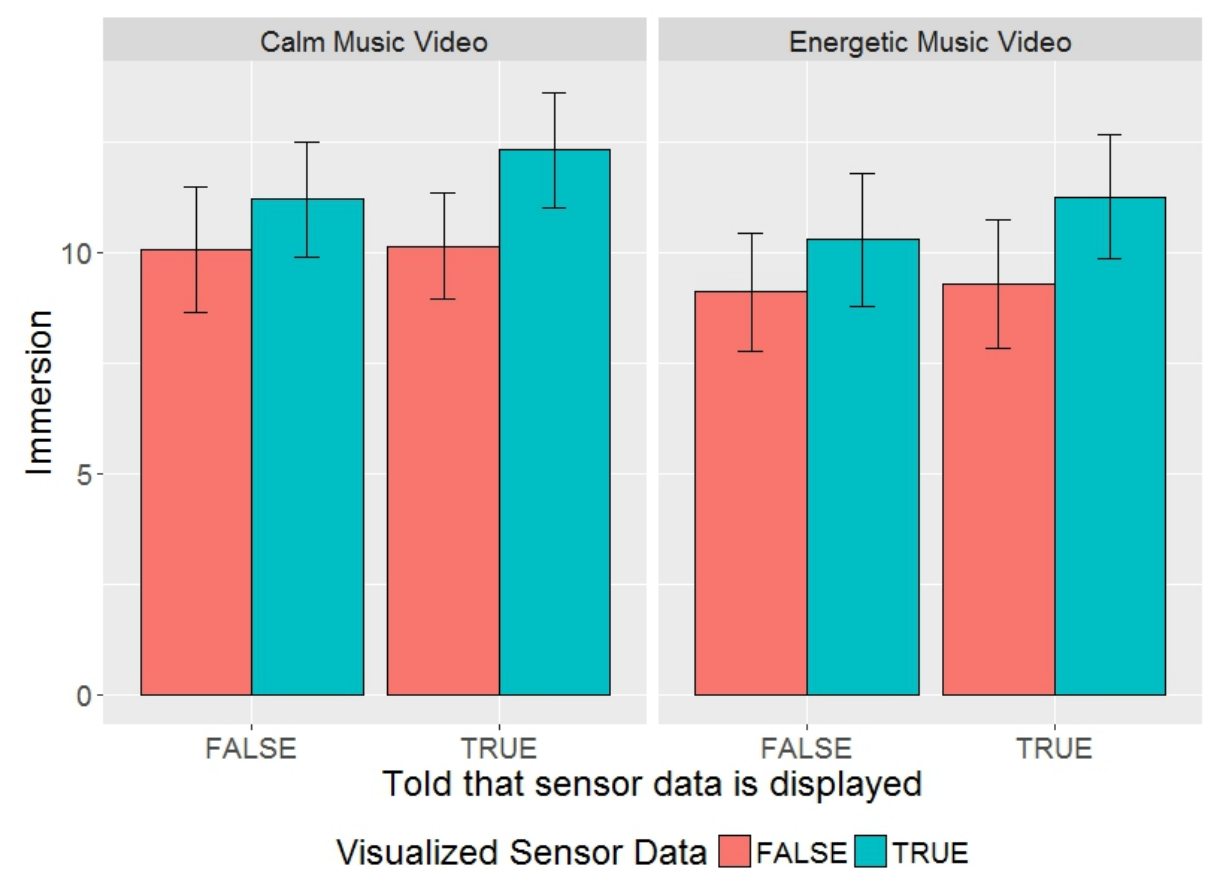

Fig. 6. Immersion levels as affected by the visualization of sensor data, the energy level in the video, and whether participants were informed or not of the presence of sensor data in the visualization

Regarding togetherness, we find similar results. In this case, however, the level of energy of the video does not significantly improve the goodness of fit of the model. As visualized in Figure 7, the presence of sensor data in the visualization is the best predictor of Togetherness (significant with $p<0.001$ ), which is higher when the sensor data are visualized. Whether participants were told that sensor data were displayed did not have an impact.

It should be noted that the togetherness values are fairly low (the maximum achievable value, having summed three items, would be 19, while on average, the togetherness feeling gets at most close to 9). This may be due to the fact that participants were shown recordings of the event, thus it was difficult for them to fully identify themselves with the audience as if they were actually attending the live event.

Finally, we found a moderate correlation between the extent to which participants liked the music excerpt played in the video and both Immersion and Togetherness (Spearman correlation coefficient of 0.41 and 0.48 , respectively). 


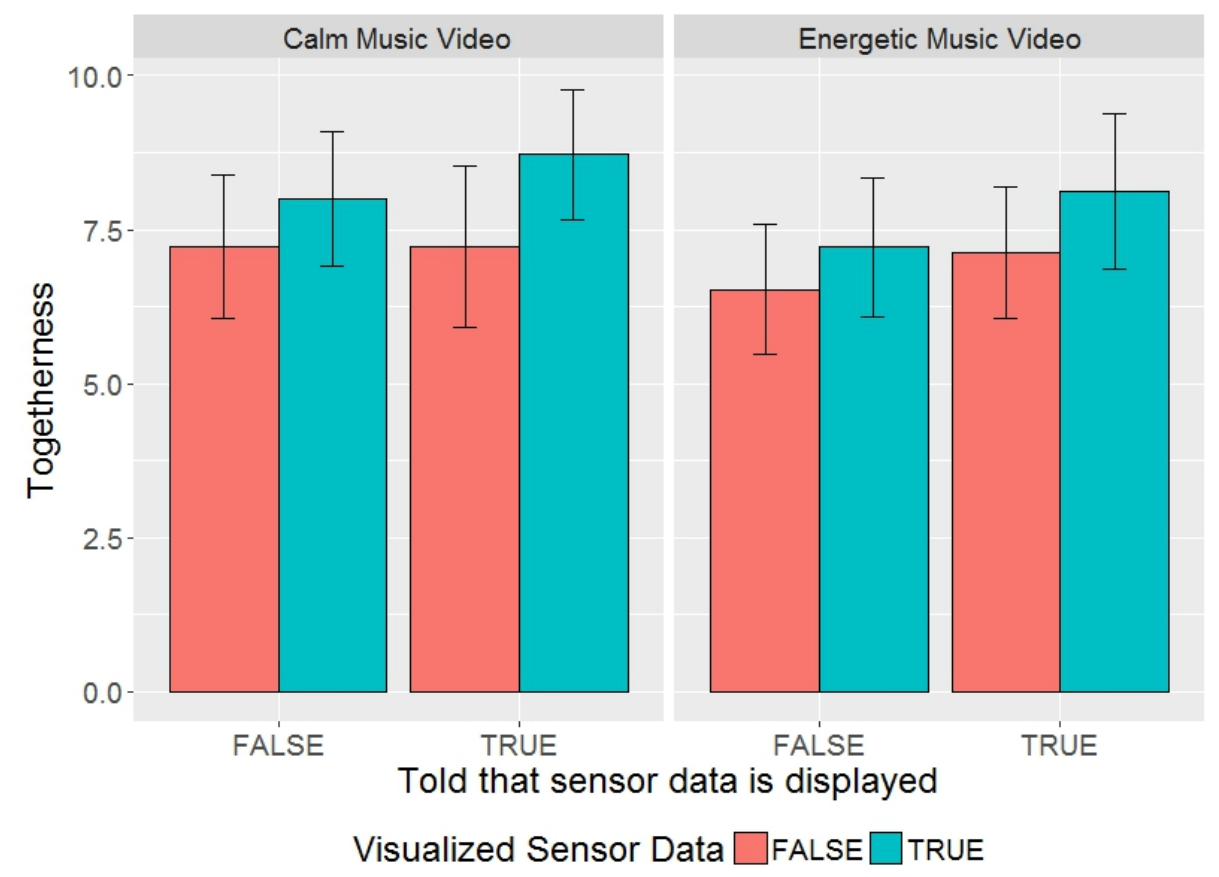

Fig. 7. Level of togetherness

This indicates that there is a tendency of participants to feel more immersed in the event and involved with the audience when they like the music better. In fact, by adding this variable as covariate in the models, we found it to have a significant effect in improving the model fit to the data, for both Immersion and Togetherness $(p<0.001$ in both cases).

\subsection{Results: Qualitative Analysis}

The qualitative evaluation confirmed that the created visualization was perceived as a 'collective artwork', which enriches the concert. The concert organizer remarked its potential added value:

"It would increase the live-experience when the audience is included in the uniqueness. Emotions are always unique - what you feel in this moment, you will never feel in this way again. This is why people go to concerts - to feel this uniqueness of the moment. And seeing this uniqueness of the moment visualized for the audience, this is an incredible added value"

Furthermore, she stated, "It is not about only the listening. When you go nowadays to a cultural event, you want to feel and experience."

As the design process was discussed, she agreed with the decision not to show the 'average mood' in the visualization, since a concert experience is "highly individual." 
"The average is not relevant, we don't need the average. [...] We live in a society where we become more and more individualized and in a 'narcissist' kind of way we want to be mirrored [in the visualization]. We all want to know more about us... And it also looks nicer!"

Finally, she highlights the collaborative aspect of a concert, which is represented in the visualization by the connecting lines between the circles (audience members).

"The atmosphere of the evening is created by the cooperation of all involved participants, also the audience, of course."

\section{Discussion and Future Research}

The main goal of the study was to determine whether a live visualization would add value to the experience of a concert. This question can be confirmed. Still, the study revealed that there are various further research areas which occur in this field of human arousal during live events. For the cultural scene, the outcomes are valuable and serve as inspiration for which purposes such visualizations could be useful and how they could be integrated into events in order to create multilayered shared experiences.

We are aware that the lab experiment set-up is not ideal as we simulated a live concert experience but are certain that it should not really affect the initial results we obtained, as we mad use of real measurements. However, as the experiments were not conducted in live concerts, the real-time algorithms to process the data are not needed for now but we used them as in the future they are necessary. Thus, we do understand that we just explored the potential experience space and got very confident results that our approach is a very good idea. It is important to mention that to be further validated, our method needs to be tested in a live scenario. Although we found well-defined, statistically significant effects of sensor data visualization on Immersion and Togetherness, the average absolute values of these two dependent variables were relatively low (around the middle of the scale, in fact). This indicates that for participants it was still difficult to fully imagine themselves in the live concert situation. Furthermore, user testing with different kinds of identifications within the visualization are needed, in order to make it easy and understandable for the concert attendee to identify herself/himself in the visualization.

The quantitative evaluation showed clearly that adding sensor data to the visualization of live music contributes to create a greater feeling of Immersion and Togetherness with the audience. Immersion was found to be influenced also by the energy level of the music played in the video excerpt: people felt more involved with the calm music video (and corresponding visualization) than with the energetic one. This result goes against our initial hypothesis that more energetic music would generate higher arousal in the audience, thereby making the visualization of sensor data more conspicuous. Also contrary to our initial hypothesis, we found that informing participants that sensor data was visualized or not did not have a significant impact on Immersion and Togetherness: 
the visualization of sensor data, rather than the knowledge of it, was most compelling for participants. Finally, we found a 'halo effect' [7] of music likability on Togetherness and Immersion. The more participants liked the music in the excerpt, the higher was their feeling of immersion and togetherness. This effect is not dissimilar from other results found e.g. in literature on visual experiences, where users more interested and engaged with the video content rated their QoE higher and were also more tolerant to impairments due to, e.g. video compression [33].

Both criteria of the collective artwork and the requirement of no evaluating the music quality were confirmed. The 'general mood' was only shown implicitly through the re-formation of the visualization; the decision to not show the average mood through a plotted line was welcomed by the concert organizer, addressing the previous concern that the visualization could be used for concert evaluation.

The visualization was developed based on requirements from the three different stakeholders in live music events: the organizer, the musician, and the audience. In order to bring the visualization closer together with the music act, it would be advisable to define and adjust the visual elements beforehand with the musicians and concert organizer into an integral and complete audio-visual concept for a deeper experience. This was also supported by the evaluation conducted with the concert organizer.

With respect to data collection, it should be noted that human arousal is a very sensitive variable, which can be triggered by various elements. Further research should be conducted to learn more about the confounding variables. In a live setting at a concert or a nightclub, the emotions of the audience are not only triggered by the music, but also by social interaction, conversations, personal thoughts; in addition, stimulants such as alcohol could influence human arousal. Further research should examine how different factors influence the GSR data and thus the visualization.

Finally, from a more practical perspective, the data was gathered, stored and pre-processed before it was used as an input for the visualization. Further work is needed to streamline these steps and make the data processing and visualization possible in real time, during a concert or at a nightclub.

\section{Conclusion}

The study presented in this paper showed that an additional visual aesthetic layer, provided by a visualization combining physiological parameters in the from of audience engagement levels and the beat and amplitude of the music, on top of the music performance during a live concert could provide a higher level of immersion of togetherness among the audience. The experiment also demonstrated that there is a tendency of participants to feel more immersed in the event and involved with the audience when they like the music better. Thus, the visualization of unique moments during the concert deepens and enriches the experience of the audience. For musicians and concert organizers, the presented 
approach of visualization is not (yet) seen as a feature in the daily concert business but rather as an experimental way towards new collaborative artworks and concert experiences. The aspect of evaluation of the event through sensor data is not welcomed neither by the musicians nor the concert organizer. Rather, it is the emotional, artistic, collaborative aspect, that appeals to the interviewed experts.

\section{References}

1. A. Aron, E. N. Aron, and D. Smollan. Inclusion of other in the self scale and the structure of interpersonal closeness. Journal of personality and social psychology, 63(4):596, 1992.

2. S. Attfield, G. Kazai, M. Lalmas, and B. Piwowarski. Towards a science of user engagement (position paper). In WSDM workshop on user modelling for Web applications, pages 9-12, 2011.

3. E. Brown and P. Cairns. A grounded investigation of game immersion. In $\mathrm{CHI}^{\prime} 04$ extended abstracts on Human factors in computing systems, pages 1297-1300. ACM, 2004

4. T. Cerratto-Pargman, C. Rossitto, and L. Barkhuus. Understanding audience participation in an interactive theater performance. In Proceedings of the 8th Nordic Conference on Human-Computer Interaction: Fun, Fast, Foundational, NordiCHI '14, pages 608-617, New York, NY, USA, 2014. ACM.

5. A. C. Davison. Statistical models, volume 11. Cambridge University Press, 2003.

6. A. Dekker. Synaesthetic performance in the club scene. In 3rd Conference on Computational Semiotics for Games and New Media, page 24, 2003.

7. K. Dion, E. Berscheid, and E. Walster. What is beautiful is good. Journal of personality and social psychology, 24(3):285, 1972.

8. A. Engström, M. Esbjörnsson, and O. Juhlin. Mobile collaborative live video mixing. In Proceedings of the 10th international conference on Human computer interaction with mobile devices and services, pages 157-166. ACM, 2008.

9. A. Engström, M. Esbjörnsson, and O. Juhlin. Nighttime visual media production in club environments. In Night and darkness: Interaction after dark-Workshop, at CHI, 2008.

10. A. Engström, M. Esbjörnsson, O. Juhlin, and C. Norlin. More tv!-support for local and collaborative production and consumption of mobile tv. Adjunct Proceedings of EuroITV, pages 173-177, 2007.

11. N. J. Evans and P. A. Jarvis. The group attitude scale a measure of attraction to group. Small Group Research, 17(2):203-216, 1986.

12. M. Faulkner. VJ: Audio-Visual Art and VJ Culture. Laurence King Publishing Ltd, 2006.

13. E. T. Hall et al. The silent language, volume 3. Doubleday New York, 1959.

14. N. Haywood and P. Cairns. Engagement with an interactive museum exhibit. In People and Computers XIXThe Bigger Picture, pages 113-129. Springer, 2006.

15. C. Jacquemin, G. Gagneré, and B. Lahoz. Shedding light on shadow: Real-time interactive artworks based on cast shadows or silhouettes. In Proceedings of the 19th ACM International Conference on Multimedia, MM '11, pages 173-182, New York, NY, USA, 2011. ACM.

16. C. Jennett, A. L. Cox, P. Cairns, S. Dhoparee, A. Epps, T. Tijs, and A. Walton. Measuring and defining the experience of immersion in games. International journal of human-computer studies, 66(9):641-661, 2008. 
17. R. Kocielnik, N. Sidorova, F. M. Maggi, M. Ouwerkerk, and J. H. Westerink. Smart technologies for long-term stress monitoring at work. In Computer-Based Medical Systems (CBMS), 2013 IEEE 26th International Symposium on, pages 53-58. IEEE, 2013.

18. P. J. Lang. The emotion probe: studies of motivation and attention. American psychologist, 50(5):372, 1995.

19. C. Latulipe, E. A. Carroll, and D. Lottridge. Love, hate, arousal and engagement: exploring audience responses to performing arts. In Proceedings of the SIGCHI Conference on Human Factors in Computing Systems, pages 1845-1854. ACM, 2011.

20. C. Martella, E. Gedik, L. Cabrera-Quiros, G. Englebienne, and H. Hung. How was it?: Exploiting smartphone sensing to measure implicit audience responses to live performances. In Proceedings of the 23rd ACM International Conference on Multimedia, MM '15, pages 201-210, New York, NY, USA, 2015. ACM.

21. H. L. O'Brien and E. G. Toms. What is user engagement? a conceptual framework for defining user engagement with technology. Journal of the American Society for Information Science and Technology, 59(6):938-955, 2008.

22. R. Picard. Affective media and wearables: Surprising findings. In Proceedings of the 22Nd ACM International Conference on Multimedia, MM '14, pages 3-4, New York, NY, USA, 2014. ACM.

23. R. W. Picard. Automating the recognition of stress and emotion: From lab to real-world impact. IEEE MultiMedia, 23(3):3-7, July 2016.

24. T. Röggla, C. Wang, and P. S. César. Analysing audience response to performing events: A web platform for interactive exploration of physiological sensor data. In Proceedings of the 23rd ACM International Conference on Multimedia, MM '15, pages 749-750, New York, NY, USA, 2015. ACM.

25. J. A. Russell. Evidence of convergent validity on the dimensions of affect. Journal of personality and social psychology, 36(10):1152, 1978.

26. T. Schubert, F. Friedmann, and H. Regenbrecht. The experience of presence: Factor analytic insights. Presence, 10(3):266-281, 2001.

27. T. W. Schubert and S. Otten. Overlap of self, ingroup, and outgroup: Pictorial measures of self-categorization. Self and identity, 1(4):353-376, 2002.

28. E. W. See-To, S. Papagiannidis, and V. Cho. User experience on mobile video appreciation: How to engross users and to enhance their enjoyment in watching mobile video clips. Technological Forecasting and Social Change, 79(8):1484-1494, 2012

29. E. A. Shanken. Art and Electronic Media. Phaidon Press Limited, 2009.

30. Y.-C. Tseng, Y.-C. Huang, K.-Y. Wu, and C.-P. Chin. Dinner of luciérnaga: An interactive play with iphone app in theater. In Proceedings of the 20th ACM International Conference on Multimedia, MM '12, pages 559-568, New York, NY, USA, 2012. ACM.

31. C. Wang, E. N. Geelhoed, P. P. Stenton, and P. Cesar. Sensing a live audience. In Proceedings of the 32nd annual ACM conference on Human factors in computing systems, pages 1909-1912. ACM, 2014.

32. B. G. Witmer and M. F. Singer. Measuring presence in virtual environments. Technical report, DTIC Document, 1994.

33. Y. Zhu, I. Heynderickx, and J. A. Redi. Understanding the role of social context and user factors in video quality of experience. Computers in Human Behavior, 49:412-426, 2015. 